\title{
Recombinaison homologue chez les parasites
}

La recombinaison homologue recouvre l'ensemble des phénomènes de recombinaison entre séquences homologues d'ADN ; elle est de plus en plus utilisée pour insérer un fragment d'ADN dans un gène donné que l'on veut inactiver ou modifier [1]. Quand de l'ADN s'intègre dans le génome d'une cellule dans laquelle il a été transféré, cette intégration peut se faire au niveau d'une séquence homologue ou bien au niveau d'une séquence quelconque. La proportion des recombinaisons homologues sur le total des intégrations (homologues + au hasard) est très élevée chez la levure (jusqu'à $75 \%$ et plus), s'abaisse chez des eucaryotes un peu plus évolués comme l'amibe Dictyostelium discoidum (environ $20 \%$ ), s'effondre chez les mammifères $(0,1$ à $0,4 \%$ [2] et plus encore chez certaines plantes. La différence entre la levure et les mammiferes tient plus à l'augmentation chez ces derniers des recombinaisons aléatoires qu'à la diminution des recombinaisons homologues. Mario Capecchi, de Salt Lake City (UT, USA), l'un des pionniers de l'utilisation de cette technique chez les souris, suppose que ce phénomène pourrait refléter le besoin des organismes ayant un grand génome de réparer efficacement, par des mécanismes rapides de recombinaison au hasard, les fractures double-brins des chromosomes qui pourraient sans cela aboutir à la perte d'une quantité considérable d'information génétique. Dans un deuxième temps, les aberrations de ce rafistolage d'urgence pourraient être elles-mêmes corrigées par conversion génique, l'allèle sain servant de "donneur de séquence" $(\mathrm{m} / \mathrm{s}$ $n^{\circ} 6$, vol. 3, p. 366 et $n^{\circ}$ 2, vol. 4,
Une équipe de Harvard medical School, à Boston (MA, USA) vient maintenant de montrer que, comme chez la levure, la recombinaison homologue était extraordinairement fréquente chez Leishmania major, un protozoaire zooflagellé de la famille des trypanosomidés se comportant comme un parasite intracellulaire du système reticulo-endothélial. Le gène de la néophosphotransférase, conférant la résistance à la kanamycine chez les procaryotes et au G418 chez les eucaryotes a été inséré au sein de séquences du gène parasitaire de la dihydrofolate réductase-thymidylate synthase (gène $d h f r$ rts) par recombinaison in vitro d'ADN. Cette construction a ensuite été transférée dans le parasite par électroporation, c'està-dire par un courant électrique créant des pores membranaires. Selon la quantité d'ADN exogène transféré, la fréquence des recombinaisons homologues au sein du gène chromosomique dhfr-ts variant de $45 \%$ à $100 \%$ de tous les événements de recombinaison [3]. Un résultat du même type a été obtenu par une équipe néerlandaise d'Amsterdam sur un autre parasite de la même famille, Trypanosoma brucei, responsable de la trypanosomiase africaine [4]. L'insertion du même gène neo que précédemment dans le gène cellulaire de tubuline était l'événement de recombinaison le plus fréquent après transfert d'ADN par électroporation. Dans les deux cas, et comme cela est déjà bien connu chez la levure et chez les mammifères, le fragment à intégrer doit être linéaire. Dans le cas de l'équipe hollandaise, il a de plus été démontré que la fréquence de recombinaison était plus grande quand les régions d'homologie de l'ADN transféré avec la séquence endogène était de $5 \mathrm{kpb}$ plutôt que de $0,9 \mathrm{kpb}$. Une telle importance de la longueur des séquences homologues a été également suggérée chez les mammifères, mais n'est pas trouvée dans tous les cas.

La haute fréquence de recombinaison homologue chez les trypanosomidés, qui sont des microorganismes diploïdes, peut permettre d'inactiver les deux allèles d'un gène, comme cela se fait couramment chez la levure. Il pourrait donc être possible, par inactivation de plusieurs gènes responsables de la virulence des parasites, de parvenir à isoler des souches très atténuées qui pourraient, peut-être, constituer la base d'une solution vaccinales à ces maladies qui continuent de faire d'importants ravages chez les humains et les animaux domestiques.

A.K.

1. Lemarchandel V, Montagutelli X. La recombinaison homologue : de nouvelles perspectives pour la transgènèse. médecine/sciences $1990 ; 6: 18-29$.

2. Capecchi MR. Gene targeting : how efficient can you get? Nature $1990 ; 348: 109$.

3. Cruz A, Berveley S. Gene replacement in parasitic protozoa. Nature $1990 ; 348$ : 171-3. 4. Ten As Brook A, Ovellette M, Borst P. Targeted insertion of the neomycin phosphotransferase gene into the tubulin gene cluster of Trypanosoma brucei. Nature $1990 ; 348: 174-5$. 